\title{
Cálculo del Costo Comparativo de la Obtención de Concentrados de Boratos
}

\author{
Horacio R. Flores y María de los Á. Tinte \\ Universidad Nacional de Salta, Facultad de Ingeniería, Instituto de Beneficio de Minerales (INBEMI), \\ CONICET, Instituto de Investigación para la Industria Química (INIQUI), Avda. Bolivia 5150, \\ 4400 Salta-Argentina (e-mail: hrflores@unsa.edu.ar)
}

\begin{abstract}
Resumen
Este trabajo muestra la similitud existente entre los diagramas de flujo, balances de materia y equipos empleados en la concentración de boratos para obtener refinados y boratos de alto valor agregado. Se usa una planilla de cálculo para determinar el costo de producción para distintas condiciones de operación, a fin de determinar la oportunidad y conveniencia de cambiar el producto final, empleando la misma planta productora. Se analiza un caso particular que compara la producción de $10 \mathrm{tn} / \mathrm{hr}$ de concentrado de ulexita o de hidroboracita. Se obtiene un costo de producción por tonelada de $\mathrm{B}_{2} \mathrm{O}_{3}$ de 94.3 US dólares para la concentración de ulexita, que se reduce a 67.5 US dólares para la concentración de hidroboracita. Se concluye que el método propuesto es una herramienta que facilita el análisis económico del proceso productivo estudiado.
\end{abstract}

Palabras claves: boratos, cálculo de costos, balance de materia, concentrado de borato, planilla de cálculo

\section{Comparative Cost Calculation in the Obtaining of Borate Concentrates}

\begin{abstract}
This paper presents the similitude found between flow sheets, material balances and equipments employed in the concentration of borates, to obtain base refined borates and to obtain high added value borates. Spread sheet calculations are used to determine the production costs for different operating conditions and to analyze the opportunity and convenience of changing the final product, using the same process plant. A study case is considered in which $10 \mathrm{tn} / \mathrm{hr}$ of ulexite concentrate or the same amount of hydroboracite are produced. The cost per ton of $\mathrm{B}_{2} \mathrm{O}_{3}$ was 94.3 US dollars for the concentrated ulexite and 67.5 US dollars for concentrated hydroboracite. It is concluded that the proposed method is an useful tool for the economic analysis of the productive process considered in this study.
\end{abstract}

Keywords: borates, costs calculation, material balance, borate concentrate, spreadsheet 


\section{INTRODUCCION}

El uso (destino) de los productos del boro fija sus especificaciones técnicas, éstas definen el proceso de obtención y por ende el costo de producción (Flores, 2004). En una economía estable como la de EEUU el valor de los productos del boro consumidos durante los últimos 15 años, referidos al año 1998, osciló entre 600 y 800 u\$s/t $\mathrm{B}_{2} \mathrm{O}_{3}$, lo que significa una variación del 25 al 33\% (U.S. Geological Survey, 2005). En Argentina se suman los imprevisibles cambios de la política económica del país que influyen en el escenario económico, tanto para la faz productiva como para la comercial.

Tomando en consideración que las empresas chicas tienden a ser más innovadoras que las grandes, Miravete y Pernías (2006) presentaron un modelo econométrico para analizar el beneficio de fabricar distintos y nuevos productos con un dado equipo y analizar otras formas de organizar la producción. Con igual criterio se desarrolló un programa que calcula consumos y costos (energéticos y de mano de obra) de las operaciones y procesos empleados en la industria boratera para la obtención de concentrados, productos pirometalúrgicos y refinados. Se resalta la similitud de los diagramas de flujo y equipos empleados en la fabricación de dichos productos, lo cual permite simular en el programa un cambio de materia prima y comparar los costos de producción de dos (o más) productos distintos empleando la misma "fábrica". El programa, destinado a productores borateros que ya disponen de una planta en funcionamiento, toma información de su diagrama de flujos, balances de masa y energía, equipos y condiciones operativas. Permite: a) determinar la influencia en el costo de producción de la variación de alguno/s de sus ítems componentes (materia prima, insumos, energía eléctrica, mano de obra, combustibles) y b) analizar la oportunidad y conveniencia de fabricar otros productos que empleen el mismo esquema operativo. Como ejemplo, se comparan costos de producción de concentrados de ulexita y de hidroboracita producidos en una misma planta.

\section{METODOLOGÍA DE CÁLCULO}

Para calcular el costo de operación de una planta ya instalada es suficiente la información contenida en un diagrama simplificado de equipos, completado con datos sobre dimensiones y características, flujos, consumos energéticos y de mano de obra. Analizar la oportunidad y conveniencia de fabricar en esa misma planta otros productos del boro requerirá, además, suministrar datos específicos del proceso de obtención del producto elegido (condiciones operativas, materia prima, propiedades físicas y químicas). Se supone operación en estado estacionario, de manera que las ecuaciones modelo (típicamente ecuaciones diferenciales) se transforman en simples ecuaciones algebraicas. El beneficio de los boratos involucra tres etapas: concentración, obtención de refinados de base y obtención de boratos especiales. En general, ver Figura 1, cada etapa emplea los mismos procesos, operaciones y equipos, sin distinción ni del borato de partida ni del producto a obtener, pudiendo así representarse con un único diagrama de flujos (Flores, 2004). De esta manera:

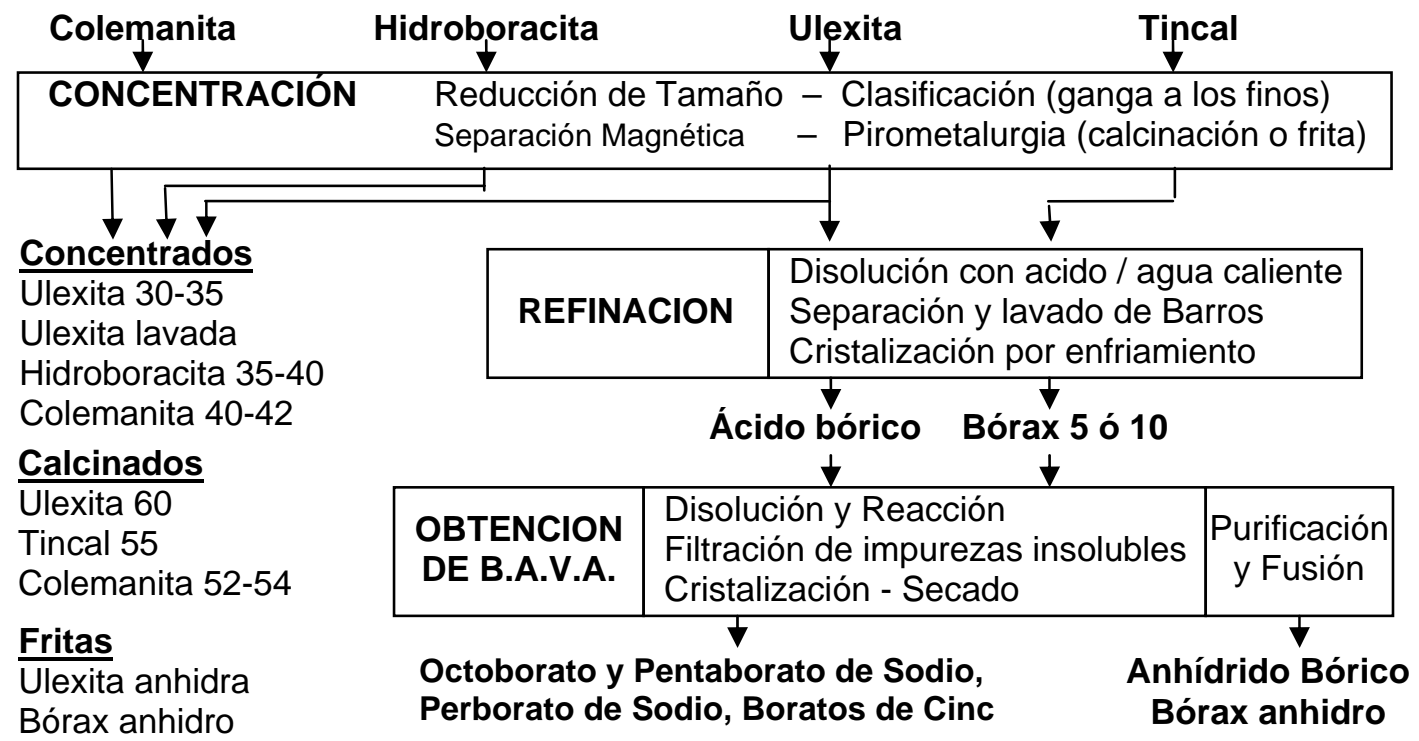

Fig. 1: Diagrama de flujos del proceso de obtención de productos del boro 
- la concentración se basa en el hecho de que los boratos, luego de someterlos a una trituración, se concentran en las fracciones de tamaño grueso. Una simple clasificación mecánica permitirá eliminar ganga en los finos. Eventualmente se emplea una separación magnética o un lavado para eliminar contaminantes de hierro o solubles (cloruros, sulfatos), respectivamente (Garret, 1998).

- la obtención de refinados de base también presentan un mismo diagrama de flujos: el ácido bórico (por disolución de ulexita con ácido sulfúrico) o el bórax (por disolución de tincal con agua caliente) implican disolución en caliente, floculación, separación y lavado de barros insolubles, cristalización por enfriamiento de la solución límpida, separación y lavado de los cristales en centrífuga y secado.

- Ios diagramas de obtención de varios productos especiales del boro (ácido bórico de alta pureza, borato de cinc, octo, penta y perborato de sodio) poseen también gran similitud (Ver Figura 1).

En el presente estudio es analizada la producción de concentrados de ulexita y de hidroboracita, basados en la información de una planta de $10 \mathrm{tn} / \mathrm{h}$ de capacidad cuyo diagrama de flujos se muestra en Figura 2. Involucra operaciones de movimiento de material con Pala Hidráulica (PH), Alimentador Vibrante (AV), Trituración en una o dos etapas (T), clasificación por tamaños en Zarandas $(Z)$, transporte en Cintas (C) y Embolsado (E). También se muestra el esquema de salida de hoja de cálculo (en Excel), indicando en las columnas los equipos correspondientes al diagrama simplificado y en las filas las dimensiones características de los equipos (ancho, altura, longitud, diámetro, volumen, potencia del motor, según corresponda), datos del material (tamaño de partícula, flujo másico, contenido en $\mathrm{B}_{2} \mathrm{O}_{3}$ ), las condiciones operativas y datos específicos al producto (set de trituradora, luz de zarandas, granulometría y distribución de $\mathrm{B}_{2} \mathrm{O}_{3}$ por tamaños). Con esta información el programa calcula los consumos de combustible, de energía eléctrica y la mano de obra necesaria (dato suministrado por el productor) los cuales, valorizados, dan el costo operativo. Por ejemplo:

- Para el cálculo del consumo energético de cintas transportadoras se considera su largo $L(m)$, velocidad $\mathrm{v}(\mathrm{m} / \mathrm{min})$ y flujo másico $\mathrm{W}(\mathrm{t} / \mathrm{h})$. Con ellos se calcula la energía Ec1 para elevar el material a una altura $h(m)$, la energía Ec2 para transportarlo horizontalmente y la energía Ec3 para mover la cinta vacía (AIMMPE, 1985). La suma de estas tres energías da el consumo total de la cinta (kW-h).

- Para el consumo energético de trituradoras se emplea la fórmula de Bond (AIMMPE, 1985), que considera el flujo másico del material a triturar W (t/min), su tamaño medio de alimentación Dpa y de descarga $\operatorname{Dpd}(\mathrm{m})$. En el ejemplo se emplea una trituradora primaria de $7 \mathrm{HP}$, una zaranda de $2 \mathrm{HP}$, seguida de una trituradora secundaria de $5 \mathrm{HP}$ y zaranda doble de $3 \mathrm{HP}$. El índice de trabajo es de 6 kwh/tc para la ulexita y 12,5 kwh/tc para la hidroboracita (Flores et al., 2002).

\begin{tabular}{|l|l|l|l|l|l|l|l|l|l|l|l|l|l|l|l|l|l|}
\hline \\
\hline Alimentación \\
\hline del Mineral
\end{tabular}

Fig. 2: Esquema de calculo de costo operativo de concentración de boratos 
Las condiciones operativas empleadas en la planilla de cálculo fueron:

a) Para ulexita (alimentación con $D p_{\max }=127 \mathrm{~mm}$ y $\left.D p_{m}=27 \mathrm{~mm}\right)$ : set trituradora primaria: 3" (76,2 mm), luz de zaranda: $1 / 4$ " $(6,35 \mathrm{~mm})$, set trituradora secundaria $7 / 8$ " $(22,2 \mathrm{~mm})$ y zaranda doble de 3,3 y $1 \mathrm{~mm}$ b) Para Hidroboracita (alimentación con $\mathrm{Dp}_{\max }=254 \mathrm{~mm}$ y $\mathrm{Dp}_{\mathrm{m}}=120 \mathrm{~mm}$ ): set trituradora primaria: 4 " (102 mm), luz de zaranda: 3/8" $(9,5 \mathrm{~mm})$ y las mismas condiciones para la segunda etapa de trituración y clasificación que las empleadas con la ulexita.

\section{RESULTADOS Y DISCUSION}

La concentración de ulexita, en 2 etapas de trituración y clasificación mecánica, eleva la ley desde 21 a $26,1 \% \mathrm{~B}_{2} \mathrm{O}_{3}$ en la primera etapa y desde 26,1 a $33,8 \%$ en la segunda, con un costo operativo de $135,7 \mathrm{u} \$ \mathrm{~s} / \mathrm{hr}$. Con el mismo esquema, la concentración de $10 \mathrm{tn} / \mathrm{hr}$ de hidroboracita eleva la ley desde 27,7 a $34,2 \% \mathrm{~B}_{2} \mathrm{O}_{3}$ en la primer etapa con un costo operativo de 74,9 u\$s/hr. Alimentar a la segunda etapa de trituración y clasificación mecánica un material del $34,2 \% \mathrm{~B}_{2} \mathrm{O}_{3}$ no es conveniente, pues se logra un concentrado del 35,3 \% $\mathrm{B}_{2} \mathrm{O}_{3}$ (sólo 1,1\% de incremento) con baja recuperación (del $42,9 \%)$ y mucho mayor costo operativo (136,3 u\$s/tn). En la Tabla 1 se comparan los resultados.

Tabla 1: Resultados comparativos del cálculo del costo operativo de concentración de boratos

\begin{tabular}{llll}
\hline Concentración de una mena de: & Ulexita & Hidroboracita & Hidroboracita \\
\hline $\mathrm{N}^{0}$ de etapas de trituración-clasificación: & 2 & 2 & 1 \\
Alimentación: Flujo másico, tn / hr & 10 & 10 & 10 \\
$\quad$ Ley $\mathrm{B}_{2} \mathrm{O}_{3}, \%$ & 21 & 27,7 & 27,7 \\
Concentrado: Flujo másico, tn / hr & 3,6 & 3,4 & 5,9 \\
$\quad L e y \mathrm{~B}_{2} \mathrm{O}_{3}, \%$ & 33,8 & 35,3 & 34,2 \\
Recuperación de $\mathrm{B}_{2} \mathrm{O}_{3}$ en el concentrado, \% & 57,9 & 42,9 & 72,8 \\
Consumo energético: Eléctrico, kWh & 13,8 & 20,3 & 9,5 \\
$\quad$ Combustible, Its Gas Oil / hr & 23,5 & 24,0 & 21,5 \\
Mano de obra, hs hombre & 29,5 & 29,5 & 15,2 \\
Costo de operación, u\$s / hr & 135,7 & 136,3 & 74,9 \\
Costo u\$s / tn $\mathrm{B}_{2} \mathrm{O}_{3}$ en el concentrado & 94,3 & 67,5 & 37,1 \\
\hline
\end{tabular}

\section{CONCLUSIONES}

Aprovechando la similitud existente entre los diagramas de flujo y los equipos empleados en la obtención de productos del boro, el programa propuesto es una herramienta que facilita el análisis económico del proceso productivo. El cálculo de costos operativos permite obtener información rápida y confiable para la toma de decisiones empresariales relativas a la influencia de insumos, materias primas y hasta proponer el cambio del producto final obtenido.

\section{REFERENCIAS}

AIMMPE, American Institute of Mining, Metallurgical and Petroleum Engineers, Inc. SME Mineral Processing Handbook. Vol. 1, pp 3B-50, 10-32, Weiss N. Editor. New York, USA (1985)

Flores H.R. El Beneficio de los Boratos, pp 130-134, 147-150, 209. Ed. Crisol. Salta-Argentina (2004)

Flores H.R., L.E. Mattenella y S.K. Valdez; Propiedades físicas y físico-químicas de boratos de la Puna Argentina. Información Tecnológica: 13 (1), 103-108 (2002)

Garret D.; "Borates". Handbook, pp 360, Academic Press. San Diego, USA (1998)

Miravete, E.J. y J.C. Pernías; Innovation Complementarity and Scale of Production. The Journal of Industrial Economics: 54 (1), 1-30 (2006)

U. S. Geological Survey; Boron. Historical Statistics for Mineral and Material Commodities in the U.S. Compiled by D.A. Buckingham and P.A. Lyday. http://minerals.usgs.gov/ds/2005/140. Acceso $25 / 5 / 2007$ 